\title{
Initiative for Developing Evidence-based Standardization of Traditional Chinese Medical Therapy in the Western Pacific Region of the World Health Organization
}

\author{
May 27-29, 2004, Seoul Korea
}

\section{II-Moo Chang}

\author{
WHO Collaborating Center for Traditional Medicines Research, Natural Products Research Institute, Seoul
}

National University, Seoul 110-460, Korea

\section{Background}

In recognition of the potential benefits of traditional medicine to the world's population, the World Health Organization (WHO) established the Traditional Medicine Program under the motto of 'Health for All by the Year of 2000' over three decades ago (The Declaration of Alma-Ata resolved from the International Conference on Primary Health Care by WHOUNICEF in Alma-Ata, USSR in 1978). Since then, WHO itself has formulated numerous projects with traditional medicines, and urged its Member States to initiate their own programs and subsequent implementation on the national level of health care programs and systems. To promote and facilitate such polices in the Western Pacific Region of the WHO, where China, Japan, Korea and Vietnam, Australia, New Zealand, Singapore and many island countries scattered in the Pacific Ocean are located, the Regional Office designated the WHO Collaboration Centers for Traditional Medicine Research in China, Japan and Korea to provide international cooperation among countries with interest in Traditional Chinese Medicine (TCM). TCM does not necessarily represent the traditional medicine of the People's Republic of China, rather the comprehensive traditional medicines of China, Japan, Korea and Vietnam, and other countries whose peoples have commonly shared in the historical development of TCM and still include some traditional medical therapies in their medical care system.

\section{The Meeting Brought Together Many Nations}

The meeting was held at the WHO Collaborating Center which has been located in the Natural Products Research Institute,

For reprints and all correspondence: II-Moo Chang. WHO Collaborating Center for Traditional Medicines Research, Natural Products Research Institute,

Seoul National University, Seoul 110-460, Korea E-mail: changim@snu.ac.kr
Seoul National University, Seoul, Republic of Korea (hereafter NPRI Center) since its designation in 1979. This institution facilitated two major initiatives for providing global standardization of TCM: (i) a proposal for establishment of the Forum on Harmonization of Herbal Medicines; and (ii) a proposal for development of the Treatment Guideline on Evidence-based Traditional Medicine.

Following the agenda on the quality standard of herbal materials and preparations, an epoch on the milestone of modernizing TCM therapy, an initiative was introduced for establishing guidelines of standard therapy practiced in TCM. There have been two serious stumbling blocks; one is a lack of standard diagnostic methodology for various diseases and syndromes in TCM practice. Therefore, each TCM doctor can diagnose subjectively with disease and syndromes. Consequently, poorly repeatable diagnoses can be made. Such a lack of standard methodology may produce a 'trial and error' therapy in practice. In this situation, one can hardly expect a standard guideline for TCM therapy. In order to work out these problems, the NPRI Center proposed that the Regional Office should formally invite an expert working group meeting and discuss issues on examining the feasibility of establishing a standard guideline for TCM therapy. Immediate action was taken by the Regional Office, and the expert meeting with the title of 'The Informal Consultation on the Development of Treatment Guidelines on Evidence-based Traditional Medicine' organized by the Regional Office and the NPRI Center was held in Seoul on May 27-29, 2004. Experts from TCM and complementary and alternative medicine (CAM), regulatory agencies, conventional medical (Western medicine) and traditional medical educational institutes, governmental policy makers from China, Korea, Japan, Taiwan, the USA and a special group of editorial staff members from $e C A M$ gathered and produced the following outcome, summarized as follows.

The online version of this article has been published under an open access model. Users are entitled to use, reproduce, disseminate, or display the open access version of this article provided that: the original authorship is properly and fully attributed; the Journal and Oxford University Press are attributed as the original place of publication with the correct citation details given; if an article is subsequently reproduced or disseminated not in its entirety but only in part or as a derivative work this must be clearly indicated. 


\section{Diseases of High Priority}

To develop treatment guidelines for evidence-based TM, participants from China, Japan and Korea submitted lists of target diseases based on their own TM development strategies. Since all countries were not represented, a list of diseases was submitted by the Regional Office to include the whole western Pacific region.

\section{China}

- Hemorrhagic stroke

- Ischemic stroke

- Cardiovascular disease

- Coronary disease

- Acute myocardial infarction

- Acute coronary syndrome

- Hypertension

- Metabolic syndromes (hypertension, hyperlipidemia, diabetes, obesity, etc.)

- Hepatitis (especially hepatitis B)

- Cancer (lung, liver, prostate, bladder)

- Influenza

- Pain syndromes (including headache, migraine, shoulder pain, back pain, etc.)

- Facial paralysis

- Pediatric diseases (infant diarrhea)

- Respiratory diseases (pneumonia)

- Parkinson's disease

- Pediatric diseases (nocturia, atopic dermatitis, epilepsy)

- Dementia

- AIDS

- Drug dependence

- Digestive diseases

- Osteoporosis

\section{Japan}

- Allergies

- Hypertension

- Coronary disease

- Cancer

- Metabolic diseases (including diabetes)

- Senile diseases (dementia)

- Pediatric diseases (atopic dermatitis)

- Psychological depression

\section{Korea}

- Hepatitis (B, C)

- Cardiovascular disease (including myocardial infarction, coronary disease, hypertension, hypotension)

- Gastrointestinal disease (including gastritis, peptic ulcer, Crohn's disease, irritable bowel syndrome)

- Respiratory diseases (chronic asthma, bronchitis)

- Influenza
- Metabolic (diabetes, thyroid diseases, obesity, hypercholesterol, high uric acid, osteoporosis)

- Musculoskeletal diseases (HIVD, ankylosing spondylitis, arthritis)

- Brain and neurological disorders (stroke, Parkinson's disease, multiple sclerosis)

- Peripheral neuropathy, facial paralysis, myasthenia gravis, muscular dystrophy, ALS

- EENT disorders (glaucoma, tinnitus, rhinitis, cataract)

- Skin diseases

- Gynecological diseases (dysmenorrhea, post-menopausal syndrome).

- Pediatric diseases (nocturia, atopic dermatitis, epilepsy, infant diarrhea)

- Neuropsychiatric syndrome (depression, dementia, neurosis)

- Cancer

- Allergy

- Pain syndromes (including headache, migraine, shoulder pain, back pain, etc.)

- AIDS

- Drug dependence

\section{The whole Pacific Region submitted by the Regional Office}

- Hepatitis (B)

- Cardiovascular disease (including myocardial infarction, coronary disease, hypertension)

- Gastrointestinal disease (including gastritis, peptic ulcer)

- Respiratory diseases (chronic asthma, bronchitis)

- Influenza

- Metabolic (diabetes, thyroid diseases, obesity, hypercholesterol, high uric acid, osteoporosis)

- Musculoskeletal diseases (HIVD, ankylosing spondylitis, arthritis)

- Brain and neurological disorders (stroke, Parkinson's disease, multiple sclerosis)

- Peripheral neuropathy, facial paralysis, myasthenia gravis, muscular dystrophy, ALS

- EENT disorders (glaucoma, tinnitus, rhinitis, cataract)

- Skin diseases

- Gynecological diseases (dysmenorrhea, post-menopausal syndrome)

- Pediatric diseases (nocturia, atopic dermatitis, epilepsy, infant diarrhea)

- Neuropsychiatric syndrome (depression, dementia, neurosis)

- Cancer

- Allergy

- Pain syndromes (including headache, migraine, shoulder pain, back pain, etc.)

- AIDS

- Drug dependence 


\section{The Process of Developing Treatment Guidelines for Evidence-Based Traditional Medicine}

\section{Purpose}

I. To pursue evidence-based scientific assessment

A. The content of evidence

B. The quality of evidence

II. Standardization of clinical practice
A. For achievement of reliability
B. For achievement of reproducibility

\section{The Process}

I. Selection of Guideline topics

A. Specific diseases

(i) In terms of traditional classification

(ii) In terms of modern classification

B. Rationale for using traditional medicine

(i) Limited availability and efficacy of modern (Western, orthodox) therapy

(ii) Socioeconomic factors (high cost of modern therapies)

(iii) Urgency and benefits of evaluating new treatments derived from traditional medical therapy

Based on the above recommendation, the Regional Office took prompt action as the first step to organize an expert working group meeting in Beijing in October, 2004, on common TCM terminology that is essential for developing the Guidelines.

CAM has evolved as a part of conventional medicine over the past decade. TCM is playing an important role in CAM, and it is an urgent task to develop a global standard of TCM practice and herbal medicines as well as for mutual recognition and understanding. To achieve the goal, efforts have been made to push forward with the standardization of TCM by international collaboration through the WHO's activities.

II. Formation of an Expert Team (Committee) by WHO Western Pacific Regional Office

A. Regional base

(i) China, Japan, and Korea

(ii) Other Member States

B. Formation

(i) Expert clinicians, basic scientists (pharmacologists, toxicologists, epidemiologists, and others, if needed), information scientists for literature review, and experts from regulatory agencies and non-governmental organizations

(ii) Formation of subcommittees for specific purposes

C. Function

(i) Develop key clinical questions

(ii) Review literature: traditional medical classics, modern scientific reports and relevant databases

(iii) Draft the Guidelines
III. Peer review

A. Extensive review of the drafted Guidelines

(i) Clinical specialists

(ii) Regional associations and clinicians' organizations

(iii) Regulatory agencies

B. Points of review

(i) Accuracy and quality

(ii) Thoroughness of drafted Guidelines

IV. Approval by the WHO Western Pacific Regional Office

A. Publication of the monographs

B. Recommendation

(i) Dissemination

(ii) Implementation of guidelines

(iii) Periodic revision of guidelines

\section{Processing the Guidelines}

I. Key clinical questions should comprise the following components:

A. TM diagnosis for specific illness (modern diagnosis is desirable)

B. Planning of therapy based on diagnosis; expected rationale and end-points of efficacy before treatment (acupuncture, moxibustion and herbal formulae)

C. Variation of treatments; rationale and end-points of efficacy changed during therapy

D. Final outcomes should include the following:

(i) Statistical analysis indicating definite efficacy

(ii) Modern assay methods that measure end-points during therapy

(iii) Benefits compared with modern therapy

II. Review of the literature

A. Searching traditional medical classics for the origin of the therapy

(i) Bibliographic studies (Chinese, Japanese, Korean and others)

(ii) Searching of relevant databases (TradiMed, TCMLAS and others)

B. Modern literature related to the diseases

(i) Bibliographic studies on various therapies

(ii) Searching databases (Medline, Napralert, STN, Agricola, Cochrane, and others)

(iii) Information on herbal formulae as pharmaceutical preparations registered to regional regulatory agencies

C. Review of abstracts and full texts

(i) Review of abstracts; determination of total number and significance of publications

(ii) Selection of information (classic and modern articles) for detailed study based on quality of literature

(iii) Review of full texts; determination of total number and significance of publications. Quality of texts will be evaluated using the following parameters:

(a) Well-controlled clinical trials applying Good Clinical Practice (GCP).

(b) Clinical papers based on case reports. 
(c) Availability of non-clinical data (Good Laboratory Practice) with respect to the efficacy and the safety of herbal formulae

(d) Acupuncture and moxibustion practiced in accordance with standardized methods

D. Assessment of the literature review: does it support the development of Guidelines?

(i) Yes

(ii) No (more data needed)

III. Finalize the Guidelines

A. A task-force team/subcommittee will be formed

(i) Data will be collected and evaluated

(ii) Data will be formulated into a Guideline with specific recommendations that ensure efficacy and safety of treatments.

B. The Guidelines will include:

(i) A title

(ii) An introduction that outlines the objectives of key clinical questions

(iii) Bibliographic sources

(iv) A summary of evidence relevant to the key clinical questions

(v) Recommendations for practice (vi) WHO disclaimer

(vii) Identification of possible gaps in the current Guidelines and directions for future research.

\section{Recommendations}

1. WHO should promote the proper use of Traditional Medicine according to the Guidelines.

2. Member states should assist with clinical trials to develop treatment guidelines.

3. The development of the Guidelines should be multidisciplinary, multinational and multicentered.

4. The WHO should harmonize the differences in preparation methods of traditional drugs in the different Member States.

5. WHO should conduct training sessions on the principles of evidence-based traditional medicine.

6. The WHO should support the preparation of the Guidelines to promote the integration of traditional medicine with modern medicine for the benefit of mankind.

Fee copies of 'The Draft Report on the Development of Treatment Guideline on Evidence-based Traditional Medicine' are available on request.

\section{FHH Standing Committee and Conference September 20-21, Shanghai, China}

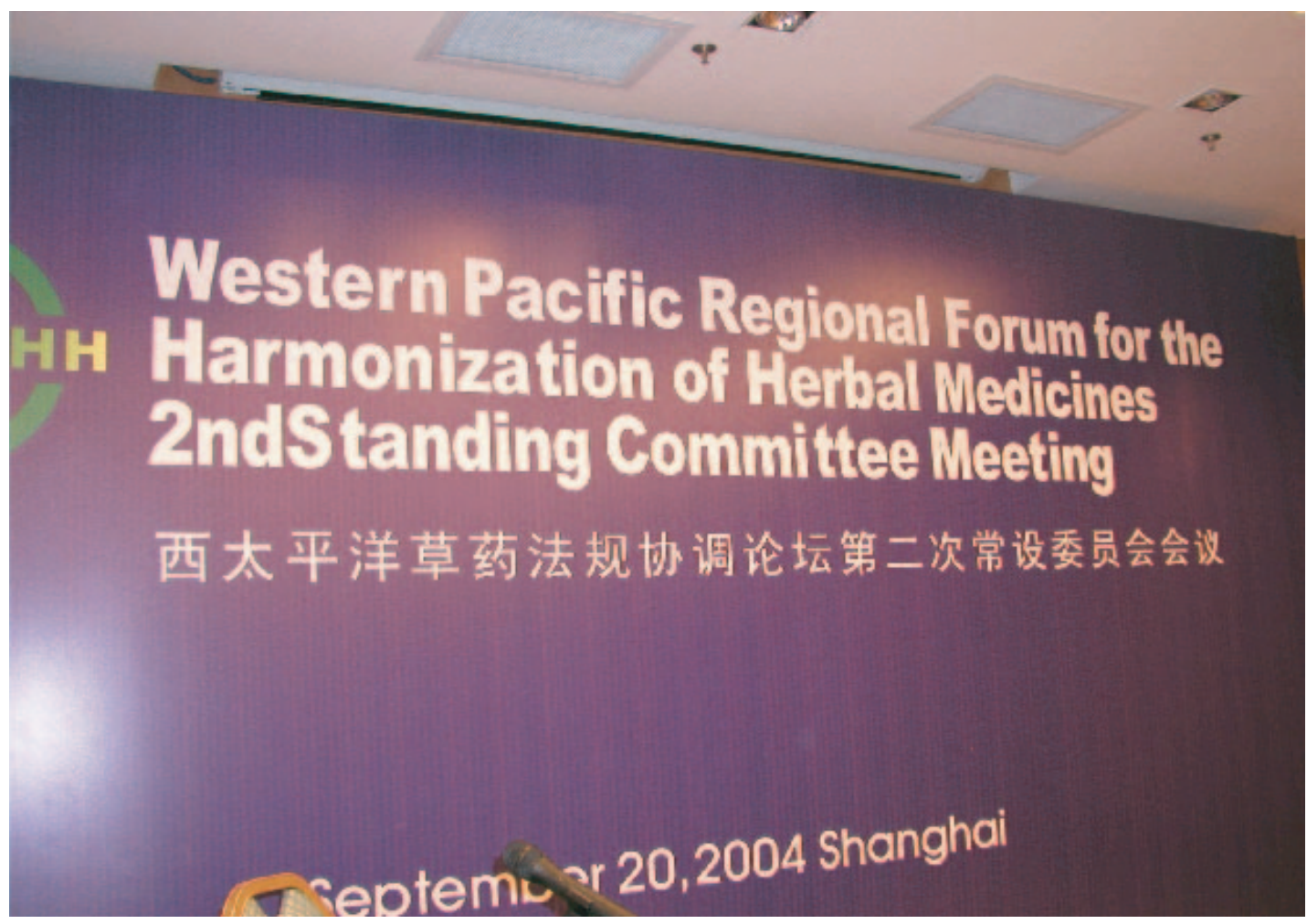

Figure 1. FHH's standing committee and conference meeting. 


\section{W.H.O. Working Group Meeting on Development of Treatment Guideline on Evidence-based Traditional Medicine May 27-28, Seoul, Korea}

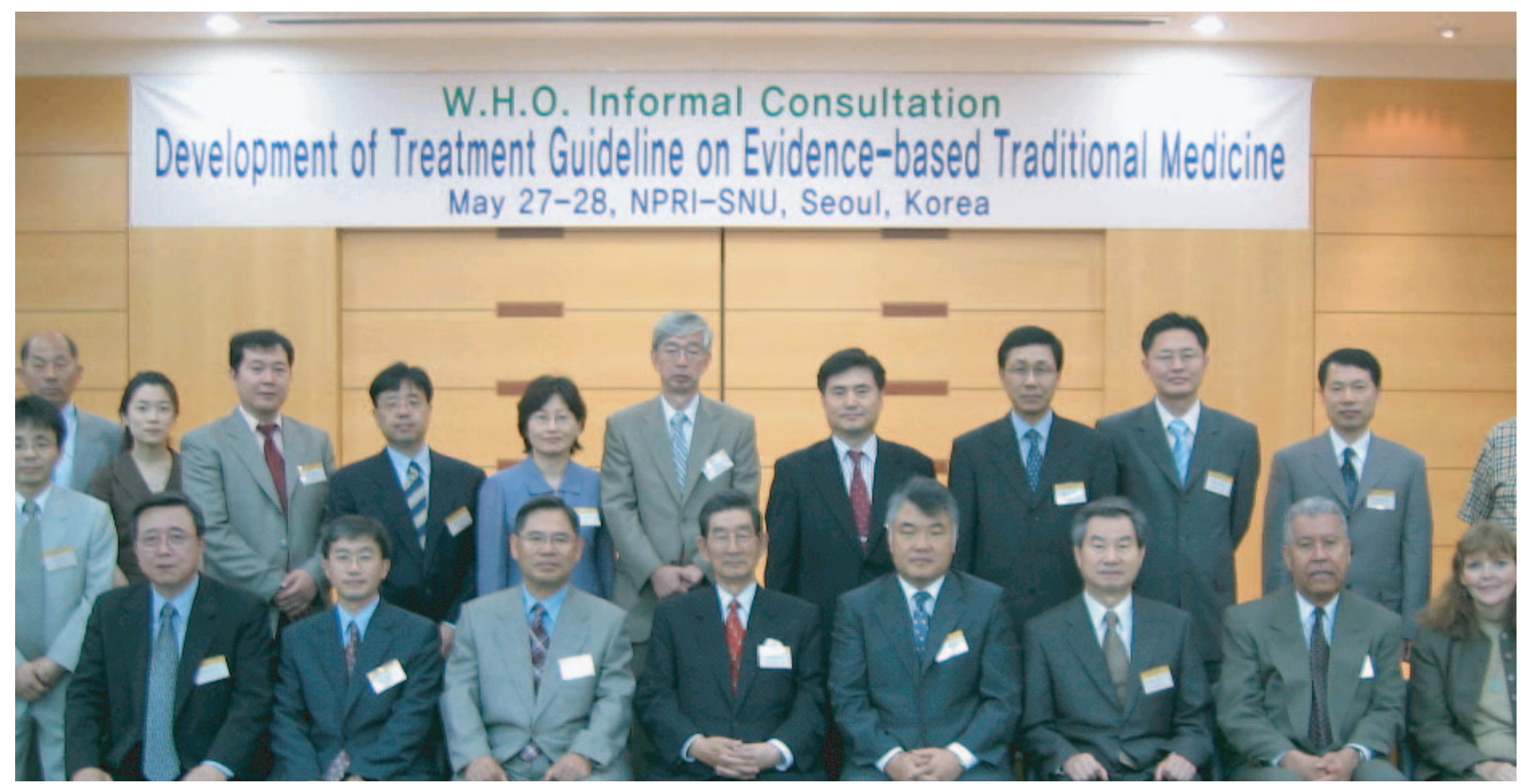

Figure 2. WHO Informal Consultation Meeting on Development of Treatment Guideline on Traditional Medicine. 


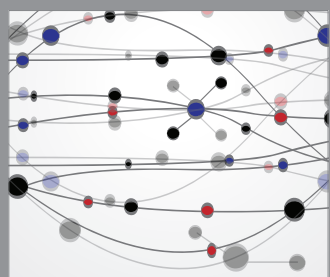

The Scientific World Journal
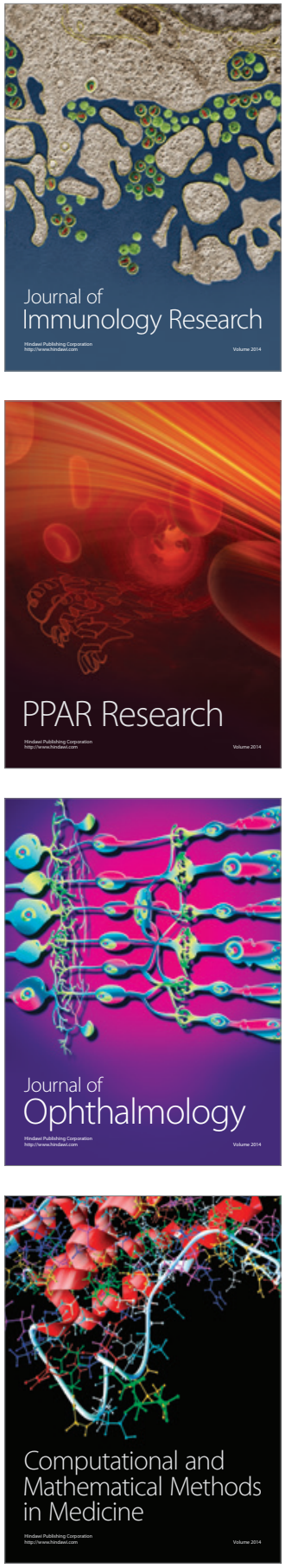

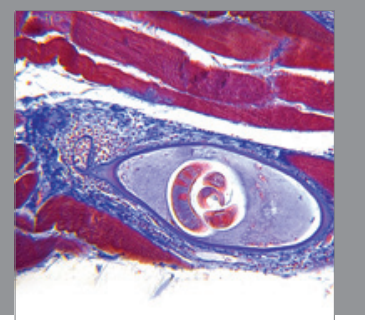

Gastroenterology

Research and Practice
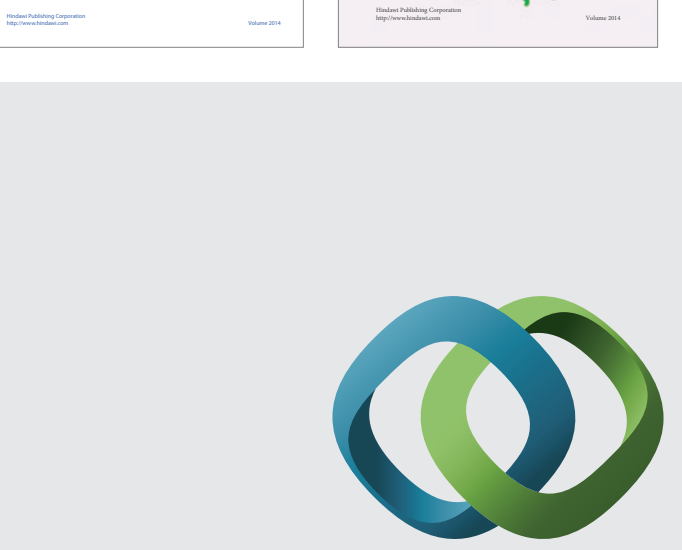

\section{Hindawi}

Submit your manuscripts at

http://www.hindawi.com
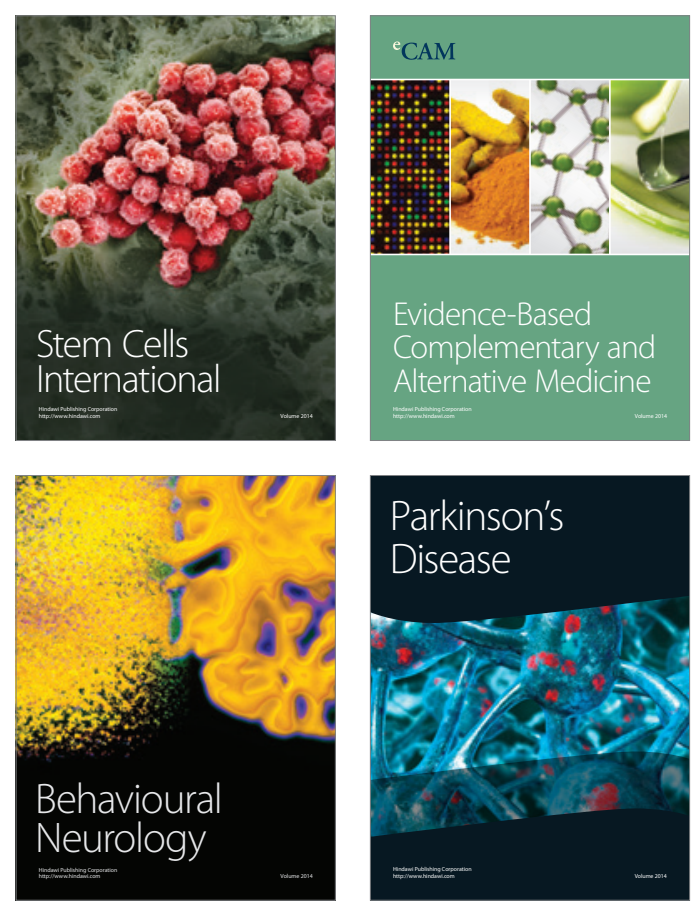

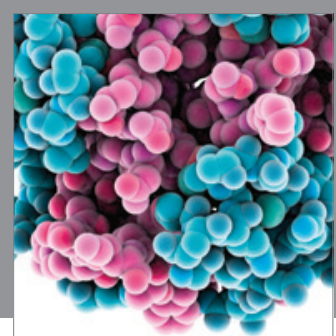

Journal of
Diabetes Research

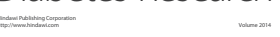

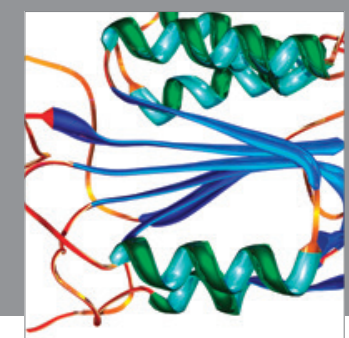

Disease Markers
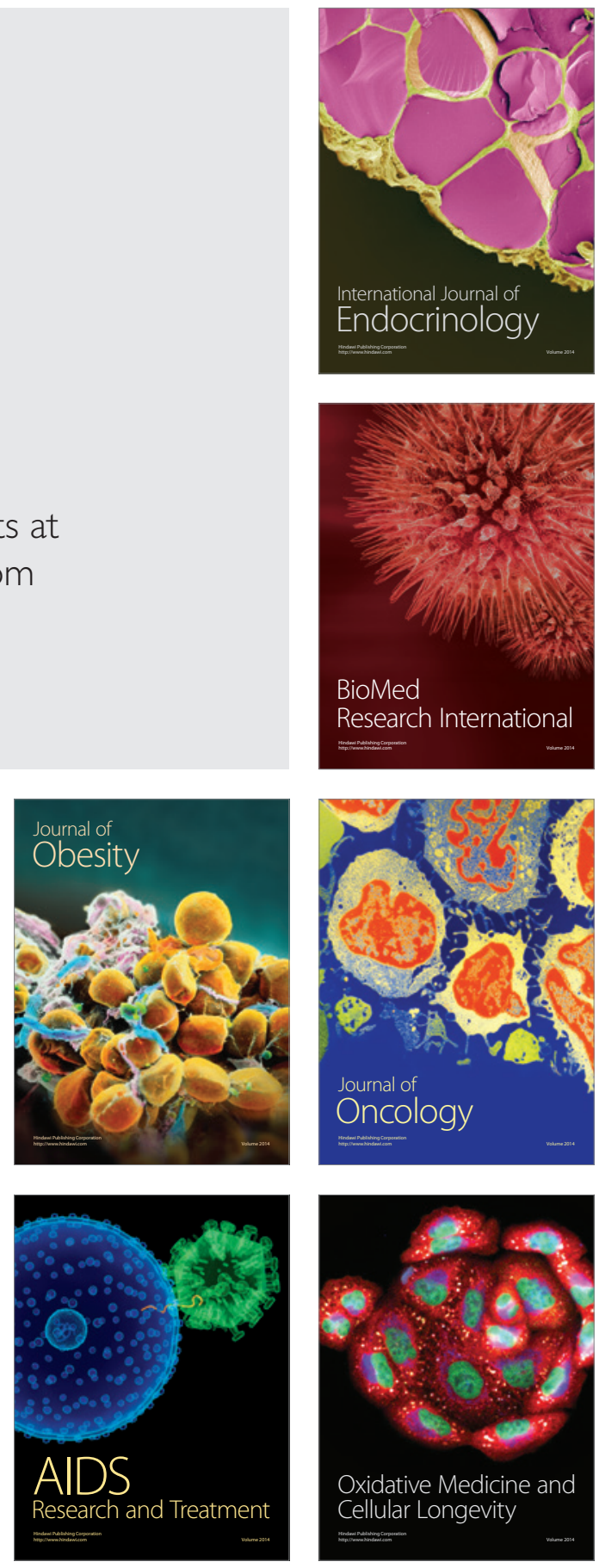